\title{
Depressão e ansiedade em profissionais de enfermagem durante a pandemia da covid-19
}

\author{
Depression and anxiety in nursing professionals during the covid-19 pandemic \\ Depresión y ansiedad en profesionales de enfermería durante la pandemia del covid-19
}

Katarina Márcia Rodrigues dos Santos ${ }^{1}$ (D) Maria Helena Rodrigues Galvão² (D)

Sávio Marcelino Gomes² (D)

Talita Araujo de Souza ${ }^{3}$ (D)

Arthur de Almeida Medeiros ${ }^{2,4}$ (DD

Isabelle Ribeiro Barbosa ${ }^{1,2}$ (D)

1. Universidade Federal do Rio Grande do Norte, Faculdade de Ciências da Saúde do Trairí, Programa de Pós-graduação em Saúde Coletiva. Santa Cruz, RN, Brasil.

2. Universidade Federal do Rio Grande do Norte, Programa de Pós-graduação em Saúde Coletiva. Natal, RN, Brasil.

3. Universidade Federal do Rio Grande do Norte, Programa de Pós-graduação em Ciências da Saúde. Natal, RN, Brasil.

4. Universidade Federal de Mato Grosso do Sul, Instituto Integrado de Saúde. Campo Grande, MS, Brasil.

\section{RESUMO}

Objetivo: Analisar a prevalência de sintomas depressão, ansiedade e fatores associados em profissionais da equipe de enfermagem durante a pandemia da Covid-19. Métodos: Estudo seccional do tipo web survey, com 490 com profissionais de enfermagem dos serviços de média e alta complexidade em um estado do nordeste do Brasil. A associação entre os desfechos e as variáveis independentes foi através do teste de qui-quadrado de Rao-Scott e do modelo de regressão de Poisson. Resultados: A ocorrência de sintomas sugestivos de transtornos mentais (ansiedade e depressão) estava relacionada a profissionais de enfermagem do sexo feminino, cor ou raça parda, com renda mensal inferior a 5 salários mínimos que trabalhavam no setor privado, ter sintomas de Síndrome de Burnout e morar com os pais. As ocorrências foram mais acentuadas quando os serviços não apresentavam condições adequadas de trabalho, em especial para o enfrentamento da pandemia de Covid-19. Conclusão e implicações para a prática: Ações que visem à melhoria das condições de trabalho e que estimulem a prática de atividades físicas podem ser benéficas para o a manutenção e fortalecimento das condições de saúde mental dessa população.

Palavras-chave: Infecções por Coronavírus; Sofrimento Mental; Saúde Mental; Depressão; Ansiedade.

\begin{abstract}
Objective: To analyze the prevalence of symptoms of depression, anxiety and associated factors in Nursing staff during the Covid-19 pandemic. Methods: A cross-sectional web survey study, with 490 Nursing professionals from medium and high complexity services in a state in northeastern Brazil. The association between the outcomes and the independent variables was through the Rao-Scott chi-square test and the Poisson regression model. Results: The occurrence of symptoms suggestive of mental disorders (anxiety and depression) was related to female Nursing professionals, brown skin color or race, with a monthly income below 5 minimum wages and working in the private sector, having symptoms of the Burnout Syndrome and live with their parents. The occurrences were more accentuated when the services did not have adequate working conditions, especially for coping with the Covid-19 pandemic. Conclusion and implications for practice: Actions that aim to improve the working conditions and that encourage the practice of physical activities can be beneficial for the maintenance and strengthening of the mental health conditions of this population.
\end{abstract}

Keywords: Coronavirus infections; Stress Psychological; Mental Health; Depression; Anxiety.

\section{RESUMEN}

Objetivo: Analizar la prevalencia de síntomas de depresión, ansiedad y factores asociados en el personal de enfermería durante la pandemia de Covid-19. Métodos: Estudio de encuesta web seccional, con 490 profesionales de enfermería de servicios de mediana y alta complejidad en un estado del noreste de Brasil. La asociación entre los resultados y las variables independientes se realizó mediante la prueba de chi-cuadrado de Rao-Scott y el modelo de regresión de Poisson. Resultados: La ocurrencia de síntomas sugestivos de trastornos mentales (ansiedad y depresión) se relacionó con mujeres profesionales de enfermería, de color o raza morena, con ingresos mensuales inferiores a 5 salarios mínimos que trabajaban en el sector privado, presentaban síntomas de Síndrome de Burnout y vivían con los padres. Los episodios eran más acentuados cuando los servicios no contaban con las condiciones laborales adecuadas, especialmente para hacer frente a la pandemia Covid-19. Conclusión e implicaciones para la práctica: Las acciones que tengan como objetivo mejorar las condiciones laborales y que incentiven la práctica de actividades físicas pueden ser beneficiosas para el mantenimiento y fortalecimiento de las condiciones de salud mental de esta población.

Palabras clave: Infecciones por Coronavirus; Estrés Psicológico; Salud Mental; Depresión; Ansiedad.
Autor correspondente:

Talita Araujo de Souza.

E-mail: talitaaraujo23@hotmail.com

Recebido em 28/08/2020

Aprovado em 18/11/2020

DOI:https://doi.org/10.1590/2177-9465-EAN-2020-0370

Escola Anna Nery 25(spe)2021 


\section{INTRODUÇÃO}

Uma nova síndrome respiratória aguda e com potencial altamente infecciosa provocada por um novo Coronavírus (SARS-CoV-2) foi identificada em dezembro de 2019, surgindo na província de Wuhan, China. No mês de janeiro de 2020, a Organização Mundial de Saúde (OMS) declarou surto de novas infecções por Coronavírus (COVID-19) e em 11 de março de 2020, a OMS declarou a Covid-19 como uma pandemia. ${ }^{1}$

Lidar com o novo Coronavírus constituiu-se um desafio global emergente no gerenciamento de doenças infecciosas. Os sintomas mais comuns duram em média 2 a 14 dias e incluem febre, fadiga, tosse seca, mialgia e dispneia. ${ }^{2} \mathrm{Em} 22$ de julho de 2020, já se somam 14.765.256 de casos confirmados em 216 países do mundo e 612.054 mortes de acordo com a Word Health Organization. ${ }^{3}$ No Brasil, nesta mesma data já existem 2.227.514 casos confirmados, mais de 82 mil óbitos com taxa de letalidade de $3,7 \%{ }^{4}$

Até o momento, nenhum tratamento antiviral ou vacina foi explicitamente recomendado para Covid-19. Dessa forma, a aplicação de medidas preventivas para controlar a infecção por Covid-19 ainda é considerada a intervenção mais efetiva. ${ }^{5}$

Diante desse contexto, estudos anteriores demonstram que durante epidemias como SARS e Ebola, o aparecimento de uma doença súbita com risco elevado de morte, leva a um grande aumento de pressão psicológica em profissionais de saúde. ${ }^{6}$ Durante esses acontecimentos, existe um aumento da carga horária de trabalho, exaustão física, ausência de equipamento de proteção, alta transmissibilidade hospitalar e necessidade de tomadas de decisões eticamente difíceis sobre racionamento de cuidados que podem mitigar em seu bem estar físico e mental. ${ }^{7,8}$

Além desses fatores, estudos afirmam que sua resiliência pode ser mais comprometida por ter que praticar o isolamento, e refletir na perda de apoio social, por representar risco de infecção a amigos e parentes. Dessa forma, profissionais de saúde são, portanto, especialmente vulneráveis a problemas de saúde mental, incluindo medo, ansiedade, depressão e insônia. ${ }^{7,8}$ Esses profissionais são considerados emocionalmente resistentes em seu local de trabalho, porém, dentro da pandemia da Covid-19 existe um novo conjunto de padrões talvez nunca experimentado por esse grupo.

A pressão de cuidar dos pacientes se intensifica no cenário de um vírus com transmissão humano-humano e sem tratamento específico para salvar vidas, ${ }^{9}$ além disso, suas vidas estão constantemente em risco, trazendo uma verdadeira situação de perigo. Outros estressores ainda podem ser elucidados, como gravidade dos pacientes, números limitados de ventiladores mecânicos e leitos de terapia intensiva ${ }^{10} \mathrm{e}$, tais fatores podem implicar diretamente no desencadeamento de crises de ansiedade e depressão.

Sabendo que os profissionais que compõem a equipe de enfermagem atuam na linha de frente do combate à COVID-19, considerando os aspectos elucidados que podem apresentar impacto na saúde mental desses profissionais, esse estudo objetivou analisar a prevalência de sintomas depressão, ansiedade e fatores associados em profissionais da equipe de enfermagem durante a pandemia da COVID-19.

\section{MÉTODOS}

Trata-se de um estudo seccional, do tipo web-survey realizado no estado do Rio Grande do Norte (RN), situado no nordeste do Brasil, direcionado às profissionais da equipe de enfermagem que atuam nos serviços saúde de média e alta complexidade. $\mathrm{O}$ período de coleta de dados estendeu-se entre os dias 04 e 17 de junho de 2020. No primeiro dia de coleta de dados, o estado possuía 14.437 casos e 444 óbitos confirmados da doença, com uma incidência de 494 casos ao dia. ${ }^{11}$ A coleta de dados ocorreu por meio de formulários eletrônicos no Google forms enviados por e-mail e redes sociais (Whatsapp $®$, Facebook@ e Instagram $®)$.

A amostra do estudo consistiu em 490 participantes, sendo o tamanho amostral calculado por meio da plataforma Open Epi, considerando a menor prevalência estimada entre os desfechos considerados no estudo (desfecho "sintomas de depressão" prevalência de $32,6 \%),{ }^{12}$ o erro absoluto de $5 \%$ e o número de profissionais de enfermagem cadastrados no Cadastro Nacional de estabelecimentos de Saúde (CNES) totalizando 10.017 profissionais, resultando na amostra de 329 indivíduos. Com a finalidade de obter uma amostra compatível com a análise por subgrupos, acrescentou-se um percentual de $50 \%$. Os critérios de inclusão da amostra consistiram em profissionais de enfermagem que atuam nos serviços de saúde de média e alta complexidade no estado do RN, e o método de amostragem foi por conveniência através da técnica de Snowball.

As variáveis dependentes do estudo consistiram nos seguintes desfechos: prevalência de sintomas de depressão moderadamente severa ou severa segundo a versão brasileira do Patient Health Questionnaire ${ }^{13}$ e a prevalência de sintomas de ansiedade moderadamente severa ou severa segundo a General Anxiety Disorder ${ }^{14}$ para mensuração de sintomas de ansiedade. As variáveis independentes do estudo consistiram em características biológicas, características socioeconômicas e familiares, características do trabalho, práticas de autocuidado, características de saúde mental e impacto da COVID-19 na vida e trabalho.

Para a composição da variável de escala de impacto da COVID-19, foi utilizado um instrumento adaptado a partir da escala de Gois e Fidalgo, utilizada originalmente para avaliar o impacto da pandemia no contexto de médicos residentes. Estas questões estão apresentadas no Quadro 1 e seus itens foram mensurados em escala do tipo likert de 1 a 5 , em que na dimensão I, 1 significou "concordo totalmente" e 5 "discordo totalmente", enquanto na dimensão II, 1 significou "nem um pouco" e 5 "extremamente".

Optou-se pela solução de três agrupamentos, considerando a interpretação teórica e a capacidade de discriminação dos componentes de cada agrupamento. Os três grupos foram definidos a partir das características da escala de impacto, assim, estão 
Quadro 1. Descrição dos itens que compõem a escala de impacto da pandemia de COVID-19.

\begin{tabular}{|l|l|}
\hline \multicolumn{1}{|c|}{ Itens da escala } \\
\hline \multirow{5}{*}{ Dimensão I } & Procuro notícias sobre a pandemia sempre que tenho tempov \\
\cline { 2 - 3 } & Tenho medo de contrair a doença e transmitir para as pessoas que amo \\
\cline { 2 - 3 } & Tenho receio de persistir com preocupação exagerada a respeito de higiene e contato após a pandemia \\
\cline { 2 - 3 } & Estou evitando atender pacientes com COVID-19 ou suspeita do mesmo \\
\cline { 2 - 3 } & Temos um serviço preparado para atender as demandas da pandemia \\
\cline { 2 - 3 } & O serviço em que trabalho disponibiliza os EPIs \\
\cline { 2 - 3 } & Acredito que os EPIs disponibilizados são eficazes para minha proteção \\
\cline { 2 - 3 } & O serviço em que trabalho disponibiliza os EPIs em quantidade suficiente \\
\cline { 2 - 3 } & Tenho convição que serei um profissional melhor qualificado por ter vivenciado a pandemia \\
\cline { 2 - 3 } & As notícias falsas tem atrapalhado o meu processo de trabalho \\
\hline & Meu consumo de bebida alcoólica aumentou durante a pandemia \\
\cline { 2 - 3 } & Meu consumo de maconha aumentou durante a pandemia \\
\cline { 2 - 3 } & Meu consumo de tabaco aumentou durante a pandemia \\
\cline { 2 - 3 } & Meu consumo de estimulantes, como cocaína ou anfetaminas, aumentou durante a pandemia \\
\hline & Tenho sentido reações físicas, como sudorese, dificuldade para respirar, náuseas ou palpitação \\
\hline & Eu me sinto capacitado para atuar no atendimento à CoVID-19 \\
\hline & Percebo que as minhas relações sociais estão comprometidas \\
\hline
\end{tabular}

Fonte: Adaptado de Gois e Fidalgo, 2020.

caracterizados da seguinte maneira: 1) profissionais de serviços menos impactados pela pandemia;2) profissionais de serviços minimamente preparados para a pandemia, considerando os fatores organizacionais e 3) profissionais de serviços sem estrutura de trabalho para a pandemia, para tal caracterização baseou-se na disponibilidade dos equipamentos de proteção individual (EPIs) e demais instrumentos/equipamentos, instalações para assistência, distanciamento e repouso entre outros, profissionais capacitados.

Para a análise de dados, realizou-se frequência absoluta e relativa das variáveis analisadas. Para a análise bivariada, realizou-se estimação da associação entre os desfechos e as variáveis independentes através do teste de qui-quadrado de Rao-Scott e suas respectivas razões de prevalências. Realizouse modelagem multivariada, através do modelo de regressão de Poisson para cada um dos desfechos avaliados. Considerando o valor de $p>0,2$ como critério de inclusão no modelo e como critério de permanência no modelo final o valor de $p<0,05$. A variável: "Teve diagnóstico de algum transtorno mental nos últimos 12 meses" foi mantida em ambos os modelos como variável de confusão para ambos os desfechos.

Esta pesquisa foi aprovada pela Comissão Nacional de Ética em Pesquisa - CONEP, sob o CAAE no 30476120.0.0000.5568 e no do parecer 4.068.729.

\section{RESULTADOS}

Responderam ao questionário 490 profissionais, sendo 292 (59,6\%) enfermeiros e 198 (40,4\%) técnicos em enfermagem. A maior parte dos profissionais respondentes eram do sexo feminino (86,7\%), com renda entre 3 e 4 salários mínimos (35,3\%). A maior parte dos profissionais atua em contato direto com pacientes com COVID-19 (89,6\%). Acerca da saúde mental, 30,4\% dos respondentes teve diagnóstico de algum transtorno mental nos últimos 12 meses, 39,6\% (IC95\%=35,3-44,0) apresentaram sintomas de ansiedade moderadamente severa ou severa, $38,0 \%$ apresentaram sintomas de depressão moderadamente severa ou severa, a presença de sintomas da Síndrome de Burnout esteve presente em $62,4 \%$ dos profissionais. Demais características podem ser observadas na Tabela 1.

Observou-se como fatores associados à ansiedade moderadamente severa ou severa, ser pardo $(R P=1,23 ; p=0,048)$, trabalhar em vínculo empregatício privado $(R P=1,70 ; p<0,001)$ ou ter vínculo público e privado $(R P=1,68 ; p<0,001)$, ter sintomas de Síndrome de Burnout ( $\mathrm{RP}=2,07 ; \mathrm{p}<0,001)$, ser profissional de serviços sem estrutura para a pandemia $(R P=2,12 ; p<0,001)$ ou maior impacto $(R P=1,75 ; p=0,012)$. Os fatores associados à menor prevalência de ansiedade moderadamente severa ou severa foram realizar atividades mente-corpo $(R P=0,46 ; p=0,003)$ e ter o hábito de conversar com amigos e familiares $(\mathrm{RP}=0,74$; $\mathrm{p}=0,003$ ) (Tabela 2). 
Tabela 1. Características biológicas, socioeconômicas, familiares e do trabalho de profissionais de enfermagem atuantes durante a pandemia de COVID-19 no estado do Rio Grande do Norte, Brasil, 2020.

\begin{tabular}{|c|c|c|}
\hline Variáveis & $\mathbf{N}$ & $\%$ (IC 95\%) \\
\hline \multicolumn{3}{|l|}{ Características biológicas } \\
\hline \multicolumn{3}{|l|}{ Sexo } \\
\hline Feminino & 425 & $86,7(83,4-89,5)$ \\
\hline Masculino & 65 & $13,3(10,5-16,6)$ \\
\hline \multicolumn{3}{|l|}{ Idade } \\
\hline 21 a 30 anos & 150 & $30,6(26,7-34,9)$ \\
\hline 31 a 36 anos & 185 & $37,8(33,6-42,1)$ \\
\hline 37 anos ou mais & 155 & $31,6(27,7-35,9)$ \\
\hline \multicolumn{3}{|l|}{ Cor da pele } \\
\hline Branco & 237 & $48,4(43,9-52,8)$ \\
\hline Pardo & 206 & $42,0(37,7-46,5)$ \\
\hline Preto & 33 & $6,7(4,8-9,3)$ \\
\hline Amarelo & 14 & $2,9(8,2-16,7)$ \\
\hline \multicolumn{3}{|l|}{ Características socioeconômicas e familiares } \\
\hline \multicolumn{3}{|l|}{ Renda Mensal do Profissional } \\
\hline Acima de 5 salários mínimos & 102 & $20,8(17,4-24,7)$ \\
\hline 3 a 4 salários mínimos & 173 & $35,3(31,2-39,7)$ \\
\hline 1 a 2 salários mínimos & 163 & $33,3(29,2-37,6)$ \\
\hline 1 salário mínimo & 52 & $10,6(8,2-16,7)$ \\
\hline \multicolumn{3}{|l|}{ Com quem mora } \\
\hline Cônjuge ou parceiro(a) & 260 & $53,1(48,6)$ \\
\hline Família (pais e/ou irmãos) & 150 & $30,6(26,7-34,9)$ \\
\hline Outros parentes ou amigos & 35 & $7,1(5,2-9,8)$ \\
\hline Sozinho & 45 & $9,2(6,9-12,1)$ \\
\hline \multicolumn{3}{|l|}{ Possui filhos } \\
\hline Sim & 246 & $50,2(45,8-54,6)$ \\
\hline Não & 244 & $49,8(45,3-54,2)$ \\
\hline \multicolumn{3}{|l|}{ Características do trabalho } \\
\hline \multicolumn{3}{|l|}{ Categoria profissional } \\
\hline Enfermeiro & 292 & $59,6(55,2-63,87)$ \\
\hline Técnico em enfermagem & 198 & $40,4(36,1-44,8)$ \\
\hline \multicolumn{3}{|l|}{ Carga horária semanal total } \\
\hline 30 horas & 105 & $21,4(18,0-25,3)$ \\
\hline 40 horas & 180 & $36,7(32,6-41,11)$ \\
\hline 60 horas & 205 & $41,8(37,5-46,3)$ \\
\hline \multicolumn{3}{|l|}{ Tipos de vínculo } \\
\hline Público & 384 & $78,4(74,5-81,8)$ \\
\hline Privado & 63 & $12,9(10,1-16,1)$ \\
\hline Ambos & 43 & $8,8(6,5-11,6)$ \\
\hline
\end{tabular}

Fonte: Dados da pesquisa, 2020. 
Tabela 1. Continuação...

\begin{tabular}{|c|c|c|}
\hline Variáveis & $\mathbf{N}$ & $\%$ (IC 95\%) \\
\hline \multicolumn{3}{|l|}{ Atua em UTI } \\
\hline Sim & 117 & $23,9(20,3-27,9)$ \\
\hline Não & 373 & $76,1(72,1-79,7)$ \\
\hline \multicolumn{3}{|l|}{ Porte do município que atua } \\
\hline Até 50.000 habitantes & 110 & $22,4(18,9-16,4)$ \\
\hline Entre 50.001 a 100.000 habitantes & 86 & $17,6(14,4-21,2)$ \\
\hline Entre 100.001 a 900.000 habitantes & 294 & $60,0(55,6-64,3)$ \\
\hline \multicolumn{3}{|l|}{ Situação atual de trabalho } \\
\hline Sigo trabalhando normalmente & 357 & $72,9(68,7-76,6)$ \\
\hline Sou caso suspeito, provável ou diagnosticado de COVID-19 & 68 & $13,9(11,1-17,24)$ \\
\hline Fui afastado/tive a função alterada & 65 & $13,3(10,5-16,6)$ \\
\hline \multicolumn{3}{|l|}{ Teve a carga horária semanal alterada após a pandemia } \\
\hline Sim & 106 & $21,6(18,2-25,5)$ \\
\hline Não & 384 & $78,4(74,5-81,8)$ \\
\hline \multicolumn{3}{|l|}{ Atua em contato direto com pacientes com COVID-19 } \\
\hline Sim & 438 & 89,6 \\
\hline Não & 52 & $10,6(8,2-13,7)$ \\
\hline \multicolumn{3}{|l|}{ Práticas de auto cuidado } \\
\hline \multicolumn{3}{|l|}{ Tem feito psicoterapia } \\
\hline Sim & 40 & $8,2(6,0-10,9)$ \\
\hline Não & 450 & $91,8(89,0-93,9)$ \\
\hline \multicolumn{3}{|l|}{ Tem tido suporte dos colegas de trabalho } \\
\hline Sim & 174 & $35,5(31,4-39,8)$ \\
\hline Não & 316 & $64,5(60,1-68,6)$ \\
\hline \multicolumn{3}{|l|}{ Tem feito atividade física } \\
\hline Sim & 152 & $31,0(27,1-35,3)$ \\
\hline Não & 338 & $69,0(64,7-72,9)$ \\
\hline \multicolumn{3}{|l|}{ Tem conversado com familiares e amigos } \\
\hline Sim & 334 & $68,2(63,9-72,1)$ \\
\hline Não & 156 & $31,8(27,8-36,1)$ \\
\hline \multicolumn{3}{|l|}{ Tem feito atividade mente-corpo } \\
\hline Sim & 66 & $13,5(10,7-16,8)$ \\
\hline Não & 424 & $86,5(83,2-89,3)$ \\
\hline \multicolumn{3}{|l|}{ Não tem realizado práticas de auto cuidado } \\
\hline Sim & 130 & $26,5(22,8-77,2)$ \\
\hline Não & 360 & $73,5(22,8-30,6)$ \\
\hline \multicolumn{3}{|l|}{ Características de saúde mental e impacto da COVID-19 } \\
\hline \multicolumn{3}{|l|}{ Teve diagnóstico de algum transtorno mental nos últimos 12 meses } \\
\hline Sim & 149 & $30,4(26,5-34,6)$ \\
\hline Não & 341 & $69,6(65,3-73,5)$ \\
\hline
\end{tabular}

Fonte: Dados da pesquisa, 2020. 
Tabela 1. Continuação...

\begin{tabular}{|c|c|c|}
\hline Variáveis & $\mathbf{N}$ & $\%$ (IC 95\%) \\
\hline Mínima ou Moderada & 296 & $60,4(56,0-64,7)$ \\
\hline Moderadamente severa ou Severa & 194 & $39,6(35,3-44,0)$ \\
\hline \multicolumn{3}{|l|}{ Classificação da severidade da depressão } \\
\hline Mínima ou nenhuma, Leve ou Moderada & 304 & $62,0(57,6-66,2)$ \\
\hline \multicolumn{3}{|l|}{ Diagnóstico de Burnout } \\
\hline Ausência & 184 & $37,6(33,3-41,9)$ \\
\hline Presença de Burnout & 306 & $62,4(58,1-66,6)$ \\
\hline \multicolumn{3}{|l|}{ Escala de impacto de COVID-19 } \\
\hline Profissionais de serviços sem estrutura de trabalho para a pandemia & 194 & $39,6(35,3-44,0)$ \\
\hline
\end{tabular}

Fonte: Dados da pesquisa, 2020.

Tabela 2. Prevalência e fatores associados à sintomas de ansiedade moderadamente severa ou severa em profissionais de enfermagem atuantes durante a pandemia de COVID-19 no estado do Rio Grande do Norte, Brasil, 2020.

\begin{tabular}{|c|c|c|c|c|c|c|}
\hline & \multicolumn{2}{|c|}{$\begin{array}{c}\text { Ansiedade } \\
\text { moderadamente severa e } \\
\text { severa }\end{array}$} & \multicolumn{2}{|c|}{ Análise bivariada } & \multicolumn{2}{|c|}{ Modelo final } \\
\hline & $\begin{array}{l}\text { Não } \\
\text { n(\%) }\end{array}$ & $\begin{array}{l}\text { Sim } \\
\mathrm{n}(\%)\end{array}$ & $p$ & RP (IC 95\%) & $p(\mathrm{aj})$. & RP (IC 95\%) (aj.) \\
\hline \multicolumn{7}{|l|}{ Características biológicas } \\
\hline \multicolumn{7}{|l|}{ Sexo } \\
\hline Masculino & $45(69,2)$ & $20(30,8)$ & - & 1 & - & - \\
\hline Feminino & $251(59,1)$ & $174(40,9)$ & 0,226 & $1,33(0,84-2,11)$ & - & - \\
\hline \multicolumn{7}{|l|}{ Idade } \\
\hline 21 a 31 anos & $82(54,7)$ & $68(45,3)$ & - & 1 & - & - \\
\hline 31 a 36 anos & $115(62,2)$ & $70(37,8)$ & 0,288 & $0,83(0,60-1,17)$ & - & - \\
\hline 37 anos ou mais & $99(63,9)$ & $56(36,1)$ & 0,209 & $0,80(0,56-1,13)$ & & - \\
\hline \multicolumn{7}{|l|}{ Cor da pele } \\
\hline Branco & $155(65,4)$ & $82(34,6)$ & - & 1 & - & 1 \\
\hline Pardo & $113(54,9)$ & $93(45,1)$ & 0,079 & $1,30(0,97-1,76)$ & $0,048 *$ & $1,23(1,01-1,51)$ \\
\hline Preto & $18(54,5)$ & $15(45,5)$ & 0,331 & $1,31(0,76-2,28)$ & 0,453 & $1,15(0,79-1,68)$ \\
\hline Amarelo & $10(71,4)$ & $4(28,6)$ & 0,709 & $0,82(0,30-2,25)$ & 0,289 & $0,70(0,38-1,34)$ \\
\hline \multicolumn{7}{|l|}{$\begin{array}{l}\text { Características } \\
\text { socioeconômicas e } \\
\text { familiares }\end{array}$} \\
\hline \multicolumn{7}{|l|}{$\begin{array}{l}\text { Renda Mensal do } \\
\text { Profissional }\end{array}$} \\
\hline $\begin{array}{l}\text { Acima de } 5 \text { salários } \\
\text { mínimos }\end{array}$ & $67(65,7)$ & $35(34,3)$ & - & 1 & - & - \\
\hline
\end{tabular}

Teste de Hosmer-Lemeshow: Qui-quadrado 278,52, valor de p=1,0; Fonte: Dados da pesquisa, 2020. 
Tabela 2. Continuação...

\begin{tabular}{|c|c|c|c|c|c|c|}
\hline & \multicolumn{2}{|c|}{$\begin{array}{c}\text { Ansiedade } \\
\text { moderadamente severa e } \\
\text { severa }\end{array}$} & \multicolumn{2}{|c|}{ Análise bivariada } & \multicolumn{2}{|c|}{ Modelo final } \\
\hline & $\begin{array}{l}\text { Não } \\
\text { n(\%) }\end{array}$ & $\begin{array}{l}\text { Sim } \\
\mathrm{n}(\%)\end{array}$ & $p$ & RP (IC 95\%) & $p$ (aj.) & RP (IC 95\%) (aj.) \\
\hline 3 a 4 salários mínimos & $102(59,0)$ & $71(41,0)$ & 0,386 & $1,97(0,78-1,79)$ & - & - \\
\hline 1 a 2 salários mínimos & $95(58,3)$ & $68(41,7)$ & 0,348 & $1,22(0,81-1,83)$ & & - \\
\hline 1 salário mínimo & $32(61,5)$ & $20(38,5)$ & 0,684 & $1,12(0,65-1,94)$ & & - \\
\hline \multicolumn{7}{|l|}{ Com quem mora } \\
\hline Cônjuge ou parceiro(a) & $161(61,9)$ & $99(38,1)$ & - & 1 & - & - \\
\hline Família (pais e/ou irmãos) & $77(51,3)$ & $73(48,7)$ & 0,112 & $1,28(0,94-1,73)$ & - & - \\
\hline Outros parentes ou amigos & $29(82,9)$ & $6(17,1)$ & 0,058 & $0,45(0,20-1,03)$ & - & - \\
\hline Sozinho & $29(64,4)$ & $16(35,6)$ & 0,799 & $0,93(0,55-1,58)$ & - & - \\
\hline \multicolumn{7}{|l|}{ Possui filhos } \\
\hline Sim & $151(61,4)$ & $95(38,6)$ & - & 1 & - & - \\
\hline Não & $145(59,4)$ & $99(40,6)$ & 0,731 & $1,05(0,79-1,39)$ & - & - \\
\hline \multicolumn{7}{|l|}{ Características do trabalho } \\
\hline \multicolumn{7}{|l|}{ Categoria profissional } \\
\hline Enfermeiro & $183(62,7)$ & $109(37,3)$ & - & 1 & - & - \\
\hline Técnico de enfermagem & $121(61,1)$ & $77(38,9)$ & 0,430 & $0,88(0,66-1,18)$ & - & - \\
\hline \multicolumn{7}{|l|}{ Carga horária semanal total } \\
\hline 30 horas & $63(60,0)$ & $42(40,0)$ & - & 1 & & - \\
\hline 40 horas & $102(56,7)$ & $78(43,3)$ & 0,676 & $1,08(0,74-1,58)$ & & - \\
\hline 60 horas & $131(63,9)$ & $74(36,1)$ & 0,595 & $0,90(0,62-1,32)$ & & - \\
\hline \multicolumn{7}{|l|}{ Tipos de vínculo } \\
\hline Público & $249(64,8)$ & $135(35,2)$ & - & 1 & & 1 \\
\hline Privado & $29(46,0)$ & $34(54,0)$ & $0,026^{*}$ & $1,54(1,05-2,24)$ & $<0,001^{*}$ & $1,70(1,30-2,24)$ \\
\hline Ambos & $18(41,9)$ & $25(58,1)$ & $0,021^{*}$ & $1,65(1,08-2,53)$ & $<0,001^{*}$ & $1,68(1,31-2,16)$ \\
\hline \multicolumn{7}{|l|}{$\begin{array}{l}\text { Em qual nível de atenção } \\
\text { atua }\end{array}$} \\
\hline Atenção secundária & $122(64,6)$ & $67(35,4)$ & - & 1 & & - \\
\hline Atenção terciária & $84(58,7)$ & $59(41,3)$ & 0,395 & $1,16(0,82-1,65)$ & & - \\
\hline Ambos & $90(57,0)$ & $68(43,0)$ & 0,260 & $1,21(0,87-1,70)$ & & - \\
\hline \multicolumn{7}{|l|}{ Atua em UTI } \\
\hline Não & $225(60,3)$ & $148(39,7)$ & - & 1 & - & - \\
\hline Sim & $71(60,7)$ & $46(39,3)$ & 0,957 & $0,99(0,71-1,38)$ & - & - \\
\hline \multicolumn{7}{|l|}{ Porte do município que atua } \\
\hline Até 50.000 habitantes & $76(69,1)$ & $34(30,9)$ & - & 1 & - & - \\
\hline $\begin{array}{l}\text { Entre } 50.001 \text { a } 100.000 \\
\text { habitantes }\end{array}$ & $56(65,1)$ & $30(34,9)$ & 0,629 & $1,13(0,69-1,84)$ & - & - \\
\hline
\end{tabular}

Teste de Hosmer-Lemeshow: Qui-quadrado 278,52, valor de p=1,0; Fonte: Dados da pesquisa, 2020. 
Tabela 2. Continuação...

\begin{tabular}{|c|c|c|c|c|c|c|}
\hline & \multicolumn{2}{|c|}{$\begin{array}{c}\text { Ansiedade } \\
\text { moderadamente severa e } \\
\text { severa }\end{array}$} & \multicolumn{2}{|c|}{ Análise bivariada } & \multicolumn{2}{|c|}{ Modelo final } \\
\hline & $\begin{array}{l}\text { Não } \\
\text { n(\%) }\end{array}$ & $\begin{array}{l}\text { Sim } \\
\mathrm{n}(\%)\end{array}$ & $p$ & RP (IC 95\%) & $p$ (aj.) & RP (IC 95\%) (aj.) \\
\hline $\begin{array}{l}\text { Entre } 100.001 \text { a } 900.000 \\
\text { habitantes }\end{array}$ & $164(55,8)$ & $130(44,2)$ & 0,063 & $1,43(0,98-2,09)$ & - & - \\
\hline \multicolumn{7}{|l|}{ Situação atual no trabalho } \\
\hline $\begin{array}{l}\text { Sigo trabalhando } \\
\text { normalmente }\end{array}$ & $221(61,9)$ & $136(38,1)$ & - & 1 & - & - \\
\hline $\begin{array}{l}\text { Sou caso suspeito, } \\
\text { provável ou } \\
\text { diagnosticado de } \\
\text { COVID-19 }\end{array}$ & $37(54,4)$ & $31(45,6)$ & 0,367 & $1,20(0,81-1,77)$ & - & - \\
\hline $\begin{array}{l}\text { Fui afastado/tive a } \\
\text { função alterada }\end{array}$ & $31(47,7)$ & $34(52,3)$ & 0,681 & $1,09(0,72-1,65)$ & - & - \\
\hline \multicolumn{7}{|c|}{ Teve a carga horária semanal alterada após a pandemia } \\
\hline Não & $213(60,2)$ & $153(39,8)$ & - & 1 & - & - \\
\hline Sim & $65(61,3)$ & $41(38,7)$ & 0,866 & $0,97(0,69-1,37)$ & - & - \\
\hline \multicolumn{7}{|c|}{ Atua em contato direto com pacientes com COVID-19 } \\
\hline Não & $35(67,3)$ & $17(32,7)$ & - & 1 & - & - \\
\hline Sim & $261(59,6)$ & $177(40,4)$ & 0,404 & $1,24(0,75-2,03)$ & - & - \\
\hline \multicolumn{7}{|l|}{ Práticas de auto cuidado } \\
\hline \multicolumn{7}{|l|}{ Tem feito psicoterapia } \\
\hline Não & $280(62,2)$ & $170(37,8)$ & - & 1 & - & - \\
\hline Sim & $16(40,0)$ & $24(60,0)$ & $0,034^{*}$ & $1,59(1,04-2,44)$ & - & - \\
\hline \multicolumn{7}{|l|}{$\begin{array}{l}\text { Tem tido suporte dos } \\
\text { colegas de trabalho }\end{array}$} \\
\hline Não & $193(61,1)$ & $123(38,9)$ & - & 1 & - & - \\
\hline Sim & $103(59,2)$ & $71(40,8)$ & 0,752 & $1,05(0,78-1,40)$ & - & - \\
\hline \multicolumn{7}{|l|}{ Tem feito atividade física } \\
\hline Não & $187(55,3)$ & $151(44,7)$ & - & 1 & - & - \\
\hline Sim & $109(71,7)$ & $43(28,3)$ & $0,008^{*}$ & $0,63(0,45-0,89)$ & - & - \\
\hline \multicolumn{7}{|l|}{$\begin{array}{l}\text { Tem conversado com } \\
\text { familiares e amigos }\end{array}$} \\
\hline Não & $73(46,8)$ & $83(53,2)$ & - & 1 & - & 1 \\
\hline Sim & $223(66,8)$ & $111(33,2)$ & $0,001^{*}$ & $0,62(0,47-0,83)$ & 0,003 & $0,74(0,60-0,90)$ \\
\hline \multicolumn{7}{|l|}{$\begin{array}{l}\text { Tem feito atividade mente- } \\
\text { corpo }\end{array}$} \\
\hline Não & $241(56,8)$ & $183(23,2)$ & - & 1 & - & 1 \\
\hline Sim & $55(83,3)$ & $11(16,7)$ & $0,002^{*}$ & $0,39(0,21-0,70)$ & 0,003 & $0,46(0,27-0,77)$ \\
\hline $\begin{array}{l}\text { Não tem realizado práticas } \\
\text { de auto cuidado }\end{array}$ & & & & & & \\
\hline
\end{tabular}

Teste de Hosmer-Lemeshow: Qui-quadrado 278,52, valor de p=1,0; Fonte: Dados da pesquisa, 2020. 
Tabela 2. Continuação...

\begin{tabular}{|c|c|c|c|c|c|c|}
\hline & \multicolumn{2}{|c|}{$\begin{array}{c}\text { Ansiedade } \\
\text { moderadamente severa e } \\
\text { severa }\end{array}$} & \multicolumn{2}{|c|}{ Análise bivariada } & \multicolumn{2}{|c|}{ Modelo final } \\
\hline & $\begin{array}{l}\text { Não } \\
\text { n(\%) }\end{array}$ & $\begin{array}{l}\text { Sim } \\
\mathrm{n}(\%)\end{array}$ & $p$ & RP (IC 95\%) & $p$ (aj.) & RP (IC 95\%) (aj.) \\
\hline Não & $238(66,1)$ & $122(33,9)$ & - & 1 & - & - \\
\hline Sim & $58(44,6)$ & $72(55,4)$ & $0,001 *$ & $1,63(1,22-2,19)$ & - & - \\
\hline \multicolumn{7}{|c|}{ Características de saúde mental e impacto da COVID-19 } \\
\hline \multicolumn{7}{|c|}{ Teve diagnóstico de algum transtorno mental nos últimos 12 meses } \\
\hline Não & $230(67,4)$ & $111(32,6)$ & - & 1 & - & 1 \\
\hline Sim & $66(44,3)$ & $83(55,7)$ & $<0,001^{*}$ & $1,71(1,29-2,27)$ & $<0,001^{*}$ & $1,43(1,17-1,74)$ \\
\hline \multicolumn{7}{|l|}{ Diagnóstico de Burnout } \\
\hline Ausência & $149(81,0)$ & $35(19,0)$ & - & 1 & - & - \\
\hline Presença de Burnout & $147(48,0)$ & $159(52,0)$ & $<0,001^{*}$ & $2,73(1,89-3,94)$ & $<0,001^{*}$ & $2,07(1,52-2,82)$ \\
\hline \multicolumn{7}{|l|}{$\begin{array}{l}\text { Escala de impacto de } \\
\text { COVID-19 }\end{array}$} \\
\hline $\begin{array}{l}\text { Profissionais de serviços } \\
\text { menos impactado pela } \\
\text { pandemia }\end{array}$ & $65(79,3)$ & $17(20,7)$ & - & 1 & - & - \\
\hline $\begin{array}{l}\text { Profissionais de } \\
\text { serviços minimamente } \\
\text { preparados para a } \\
\text { pandemia }\end{array}$ & $143(66,8)$ & $71(33,2)$ & 0,082 & $1,60(0,94-2,72)$ & 0,091 & $1,47(0,94-2,29)$ \\
\hline $\begin{array}{l}\text { Profissionais de serviços } \\
\text { sem estrutura para a } \\
\text { pandemia }\end{array}$ & $88(45,4)$ & $106(54,6)$ & $<0,001^{*}$ & $2,63(1,58-4,40)$ & $<0,001^{*}$ & $2,12(1,39-3,22)$ \\
\hline
\end{tabular}

Teste de Hosmer-Lemeshow: Qui-quadrado 278,52, valor de p=1,0; Fonte: Dados da pesquisa, 2020.

Como fatores associados a depressão moderadamente severa ou severa, destacou-se ser do Sexo feminino $(\mathrm{RP}=1,62$; $p=0,032)$, ter renda mensal de 3 a 4 salários mínimos $(R P=1,41$; $p=0,035)$, morar com os pais e irmãos $(R P=1,32 ; p=0,012)$, atuar apenas em serviços privados ( $R P=1,57 ; p=0,002)$, ter se afastado do serviço ou ter a função alterada em decorrência da pandemia $(R P=1,35 ; p=0,012)$, ter sintomas de Síndrome de Burnout $(R P=2,16 ; p<0,001)$, ser profissional de serviços sem estrutura para a pandemia $(R P=1,82 ; p=0,002)$. Os fatores associados à menor prevalência de depressão moderadamente severa ou severa foram realizar atividades físicas $(R P=0,64$; $\mathrm{p}=0,002)$ e ter o hábito de conversar com amigos e familiares $(\mathrm{RP}=0,74 ; \mathrm{p}=0,003)$ (Tabela 3).

\section{DISCUSSÃO}

Observou-se no presente estudo, que profissionais que atuam em serviços privados, que possuem sintomas de Síndrome de Burnout, e que atuam em serviços sem estrutura para o enfrentamento da pandemia de COVID-19, possuem maiores prevalências de sintomas de ansiedade e depressão. Enquanto o hábito de ter conversas com amigos e familiares mostrou-se um fator que reduz a prevalência de sintomas de ansiedade e depressão em profissionais de enfermagem, durante a pandemia de COVID-19.

Resultados semelhantes foram observados no estudo de Que et al., ${ }^{15}$ com profissionais de saúde da China durante a pandemia de COVID-19, que identificou que quase metade dos enfermeiros entrevistados relataram sintomas de depressão, ansiedade e insônia. Também foi observado que ter uma maior renda familiar e praticar atividades físicas foram fatores protetores para os sintomas de depressão.

Os profissionais de enfermagem apresentam maior predisposição para sofrimento mental, sendo a depressão uma dentre três das doenças que mais os acometem. Isto se deve não só a natureza da atividade que desenvolvem; que está diretamente relacionada a sofrimentos físicos e emocionais daqueles a quem estes prestam seus serviços, mas também as condições de trabalho e falta de reconhecimento profissional. ${ }^{16}$ 
Tabela 3. Prevalência e fatores associados a sintomas de depressão moderadamente severa e severa em profissionais de enfermagem atuantes durante a pandemia de COVID-19 no estado do Rio Grande do Norte, Brasil, 2020.

\begin{tabular}{|c|c|c|c|c|c|c|}
\hline & \multicolumn{2}{|c|}{$\begin{array}{c}\text { Depressão } \\
\text { moderadamente severa } \\
\text { e severa }\end{array}$} & \multicolumn{2}{|c|}{ Análise bivariada } & \multicolumn{2}{|c|}{ Modelo final } \\
\hline & $\begin{array}{l}\text { Não } \\
\mathrm{n}(\%)\end{array}$ & $\begin{array}{l}\operatorname{sim} \\
n(\%)\end{array}$ & $p$ & RP (IC 95\%) & $p$ (aj.) & RP (IC 95\%) (aj.) \\
\hline \multicolumn{7}{|l|}{ Características biológicas } \\
\hline \multicolumn{7}{|l|}{ Sexo } \\
\hline Masculino & $51(78,5)$ & $14(21,5)$ & - & 1 & - & 1 \\
\hline Feminino & $253(59,5)$ & $172(40,5)$ & $0,023^{*}$ & $1,88(1,09-3,24)$ & $0,032^{*}$ & $1,62(1,04-2,52)$ \\
\hline \multicolumn{7}{|l|}{ Idade } \\
\hline 21 a 31 anos & $82(54,7)$ & $68(45,3)$ & - & 1 & - & - \\
\hline 31 a 36 anos & $122(65,9)$ & $63(34,1)$ & 0,102 & $0,75(0,53-1,06)$ & - & - \\
\hline 37 anos ou mais & $100(64,5)$ & $55(35,5)$ & 0,177 & $0,78(0,55-1,12)$ & - & - \\
\hline \multicolumn{7}{|l|}{ Cor da pele } \\
\hline Branco & $161(67,9)$ & $76(32,1)$ & - & 1 & - & - \\
\hline Pardo & $115(55,8)$ & $91(44,2)$ & $0,039 *$ & $1,38(1,02-1,87)$ & - & - \\
\hline Preto & $18(54,5)$ & $15(45,5)$ & 0,217 & $1,42(0,81-2,47)$ & - & - \\
\hline Amarelo & $10(71,4)$ & $4(28,6)$ & 0,822 & $0,89(0,33-2,44)$ & - & - \\
\hline \multicolumn{7}{|l|}{$\begin{array}{l}\text { Características socioeconômicas } \\
\text { e familiares }\end{array}$} \\
\hline \multicolumn{7}{|l|}{ Renda Mensal do Profissional } \\
\hline Acima de 5 salários mínimos & $72(70,6)$ & $30(29,4)$ & - & 1 & - & 1 \\
\hline 3 a 4 salários mínimos & $98(56,6)$ & $75(43,4)$ & 0,073 & $1,47(0,97-2,25)$ & $0,035^{*}$ & $1,41(1,02-1,94)$ \\
\hline 1 a 2 salários mínimos & $95(58,3)$ & $68(41,7)$ & 0,111 & $1,42(0,92-2,18)$ & 0,052 & $1,38(1,00-1,91)$ \\
\hline 1 salário mínimo & $39(75,0)$ & $13(25,0)$ & 0,625 & $0,85(0,44-1,63)$ & 0,913 & $0,92(0,58-1,44)$ \\
\hline \multicolumn{7}{|l|}{ Com quem mora } \\
\hline Cônjuge ou parceiro(a) & $171(65,8)$ & $89(34,2)$ & - & 1 & - & 1 \\
\hline Família (pais e/ou irmãos) & $80(53,3)$ & $70(46,7)$ & 0,052 & $1,36(1,00-1,86)$ & $0,012^{*}$ & $1,32(1,06-1,65)$ \\
\hline Outros parentes ou amigos & $26(74,3)$ & $9(25,7)$ & 0,413 & $0,75(0,38-1,49)$ & 0,514 & $0,85(0,52-1,39)$ \\
\hline Sozinho & $27(60,0)$ & $18(40,0)$ & 0,547 & $1,17(0,70-1,94)$ & 0,480 & $1,15(0,78-1,68)$ \\
\hline \multicolumn{7}{|l|}{ Possui filhos } \\
\hline Sim & $160(65,0)$ & $86(35,0)$ & - & 1 & - & - \\
\hline Não & $144(59,0)$ & $100(41,0)$ & 0,280 & $1,17(0,88-1,56)$ & - & - \\
\hline \multicolumn{7}{|l|}{ Características do trabalho } \\
\hline \multicolumn{7}{|l|}{ Categoria profissional } \\
\hline Enfermeiro & $183(62,7)$ & $109(37,3)$ & - & & - & - \\
\hline Técnico em enfermagem & $121(61,1)$ & $77(38,9)$ & 0,783 & $1,04(0,78-1,39)$ & - & - \\
\hline \multicolumn{7}{|l|}{ Carga horária semanal total } \\
\hline 30 horas & $67(63,8)$ & $38(36,2)$ & - & 1 & - & - \\
\hline 40 horas & $112(62,2)$ & $68(37,8)$ & 0,832 & $1,04(0,70-1,55)$ & - & - \\
\hline 60 horas & $125(61,0)$ & $80(39,0)$ & 0,702 & $1,08(0,73-1,59)$ & - & - \\
\hline
\end{tabular}

Teste de Hosmer-Lemeshow: Qui-quadrado 270,73; valor de $p=1,000$. Fonte: Dados da pesquisa, 2020. 
Tabela 3. Continuação...

\begin{tabular}{|c|c|c|c|c|c|c|}
\hline & \multicolumn{2}{|c|}{$\begin{array}{c}\text { Depressão } \\
\text { moderadamente severa } \\
\text { e severa } \\
\end{array}$} & \multicolumn{2}{|c|}{ Análise bivariada } & \multicolumn{2}{|c|}{ Modelo final } \\
\hline & $\begin{array}{l}\text { Não } \\
\mathrm{n}(\%)\end{array}$ & $\begin{array}{l}\text { Sim } \\
\mathrm{n}(\%)\end{array}$ & $p$ & RP (IC 95\%) & $p$ (aj.) & RP (IC 95\%) (aj.) \\
\hline \multicolumn{7}{|l|}{ Tipos de vínculo } \\
\hline Público & $245(63,8)$ & $139(36,2)$ & - & 1 & & 1 \\
\hline Privado & $33(52,4)$ & $30(47,6)$ & 0,173 & $1,32(0,89-1,95)$ & $0,002^{*}$ & $1,57(1,18-2,01)$ \\
\hline Ambos & $26(60,5)$ & $17(39,5)$ & 0,731 & $1,09(0,66-1,81)$ & 0,928 & $1,01(0,72-1,44)$ \\
\hline \multicolumn{7}{|l|}{ Em qual nível de atenção atua } \\
\hline Atenção secundária & $120(63,5)$ & $69(36,5)$ & - & 1 & - & - \\
\hline Atenção terciária & $88(61,5)$ & $55(38,5)$ & 0,773 & $1,05(0,74-1,50)$ & - & - \\
\hline Ambos & $96(60,8)$ & $62(39,2)$ & 0,680 & $1,07(0,76-1,51)$ & & \\
\hline \multicolumn{7}{|l|}{ Atua em UTI } \\
\hline Não & $232(62,2)$ & $141(37,8)$ & - & 1 & - & - \\
\hline Sim & $72(61,5)$ & $45(38,5)$ & 0,919 & $1,02(0,73-1,42)$ & - & - \\
\hline \multicolumn{7}{|l|}{ Porte do município que atua } \\
\hline Até 50.000 habitantes & $80(72,7)$ & $30(27,3)$ & - & 1 & - & - \\
\hline $\begin{array}{l}\text { Entre } 50.001 \text { a } 100.000 \\
\text { habitantes }\end{array}$ & $56(65,1)$ & $30(34,9)$ & 0,340 & $1,28(0,77-2,12)$ & - & - \\
\hline $\begin{array}{l}\text { Entre } 100.001 \text { a } 900.000 \\
\text { habitantes }\end{array}$ & $168(57,1)$ & $126(42,9)$ & $0,026^{*}$ & $1,57(1,06-2,34)$ & - & - \\
\hline \multicolumn{7}{|l|}{ Situação atual no trabalho } \\
\hline $\begin{array}{l}\text { Sigo trabalhando } \\
\text { normalmente }\end{array}$ & $236(66,1)$ & $121(33,9)$ & - & 1 & - & 1 \\
\hline $\begin{array}{l}\text { Sou caso suspeito, provável } \\
\text { ou diagnosticado de COVID-19 }\end{array}$ & $37(54,4)$ & $31(45,6)$ & 0,141 & $1,35(0,91-2,00)$ & 0,979 & $1,00(0,73-1,39)$ \\
\hline $\begin{array}{l}\text { Fui afastado/tive a função } \\
\text { alterada }\end{array}$ & $31(47,7)$ & $34(52,3)$ & $0,025 *$ & $1,54(1,05-2,26)$ & $0,012^{*}$ & $1,35(1,07-1,71)$ \\
\hline \multicolumn{7}{|c|}{ Teve a carga horária semanal alterada após a pandemia } \\
\hline Não & $239(62,2)$ & $145(37,8)$ & - & 1 & - & - \\
\hline Sim & $65(61,3)$ & $41(38,7)$ & 0,892 & $1,02(0,72-1,45)$ & - & - \\
\hline \multicolumn{7}{|c|}{ Atua em contato direto com pacientes com COVID-19 } \\
\hline Não & $36(69,2)$ & $16(30,8)$ & - & 1 & - & - \\
\hline Sim & $268(61,2)$ & $170(38,8)$ & 0,374 & $1,26(0,76-2,11)$ & - & - \\
\hline \multicolumn{7}{|l|}{ Práticas de auto cuidado } \\
\hline \multicolumn{7}{|l|}{ Tem feito psicoterapia } \\
\hline Não & $289(64,2)$ & $161(35,8)$ & - & 1 & - & - \\
\hline Sim & $15(37,5)$ & $25(62,5)$ & $0,009 *$ & $1,75(1,15-2,66)$ & - & - \\
\hline \multicolumn{7}{|l|}{$\begin{array}{l}\text { Tem tido suporte dos colegas de } \\
\text { trabalho }\end{array}$} \\
\hline Não & $196(62,0)$ & $120(38,0)$ & - & 1 & - & - \\
\hline
\end{tabular}

Teste de Hosmer-Lemeshow: Qui-quadrado 270,73; valor de $p=1,000$. Fonte: Dados da pesquisa, 2020. 
Tabela 3. Continuação...

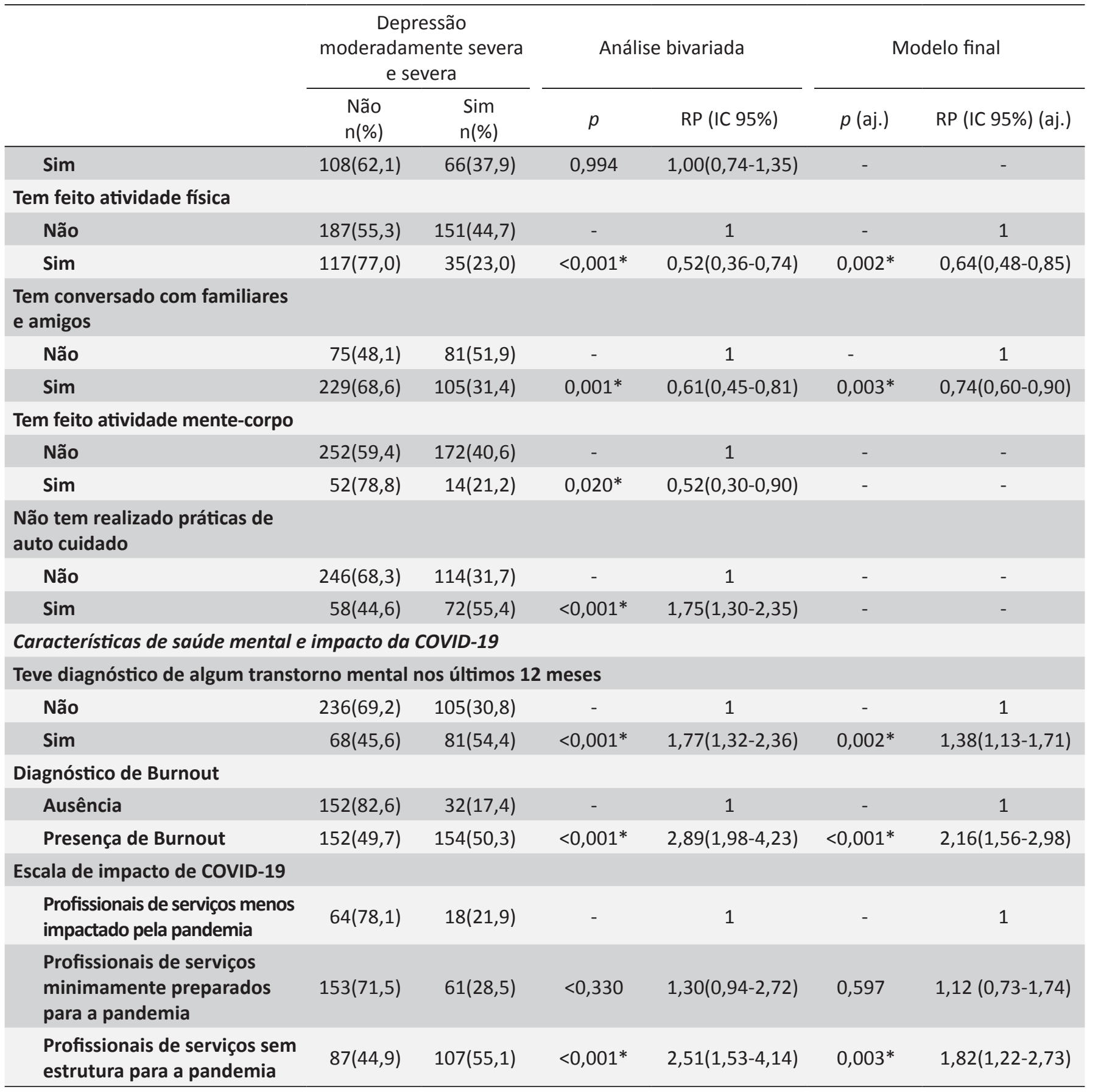

Teste de Hosmer-Lemeshow: Qui-quadrado 270,73; valor de p=1,000. Fonte: Dados da pesquisa, 2020.

Outro impacto da atual pandemia foi à adoção do distanciamento social, que provocou alterações na forma como as pessoas se relacionam. ${ }^{17}$ Ao considerar a natureza sociável dos indivíduos, que possuem necessidades de interações e que tais interações são fundamentais para construção individual, desenvolvimento, aprendizagem, ensino, criação de vínculos; o distanciamento é um fator negativo na reestruturação psíquica. ${ }^{18} \mathrm{E}$ pode gerar incertezas, apreensões, pânico, quadros de ansiedade, medo solidão, dentre outros, desencadeando sofrimento mental.

O outro fator relacionado que se destacou em relação depressão foi a renda mensal dos profissionais, onde os profissionais com renda mensal de 3 a 4 salários mínimos obtiveram uma prevalência de sintomas de depressão moderadamente severa ou severa $41 \%$ maior que profissionais com renda de mensal igual ou maior a 5 salários mínimos. Entretanto, é importante 
ressaltar que o efeito da renda não foi observado nos estratos de renda inferiores.

Sobre a renda, estudos demonstram que quanto menor a renda, maior é a prevalência de depressão, podendo se relacionar com este fato, as vidências desta prevalência em profissionais com apenas um vínculo empregatício. Entretanto, quanto mais vínculos empregatícios, maior o aumento de renda e também maior o impacto na saúde mental, devido ao desgaste profissional. ${ }^{16} \mathrm{Na}$ maioria das situações, este aumento de vínculos está relacionado à baixos salários, ausência de piso salarial, e dupla ou tripla jornada com a finalidade de aumentar os rendimentos, que são fatores que agravam ou provocam desgaste físico e psicológico. ${ }^{19}$

Ao avaliar as características do trabalho, verificou-se que a prevalência de depressão moderadamente severa ou severa entre profissionais que atuam em serviços sem estrutura para o combate a pandemia foi $86 \%$, maior que em profissionais de serviços menos impactado pela pandemia. Uma possível explicação para este achado foi o aumento circunstancial da demanda dos serviços e a escassez de insumos e pessoal, que foi agravada pelas ausências e afastamentos que ocorrem, ou por pertencer ao grupo de risco ou por adoecimento. Essa escassez provoca o sentimento de desvalorização, tornando o desgaste e o sofrimento no trabalho mais intensos. ${ }^{19}$

A influência desses fatores não se dá apenas entre os profissionais que permanecem em atividade, tendo em vista que profissionais que referiram estar afastados ou ter a função alterada devido a pandemia de COVID-19, apresentaram uma prevalência de sintomas graves de depressão 35\% maior que indivíduos que seguem trabalhando normalmente. Vale ressaltar que tais profissionais podem ter sido afastados por serem casos suspeitos ou confirmados de COVID-19, o que gera impacto na condição de saúde mental de tais profissionais. ${ }^{20}$

Têm-se observado que a estrutura do trabalho influencia no surgimento de sofrimento mental, tendo sido relacionado aos ambientes insalubres, condições precárias, conflitos internos, cobrança dos acompanhantes, falta de autonomia profissional, insegurança no desenvolvimento de suas atividades, sobrecarga de trabalho e exigências da instituição. ${ }^{16} \mathrm{~A}$ atual situação sanitária amplificou tais condições, em muitos casos os profissionais exercem suas atividades em situações de risco, com estrutura física inadequada, escassez de recursos materiais, sobrecarga de funções, carga horária extensa e falta de capacitação profissional. ${ }^{19}$

Em decorrência disso, o Conselho Federal de Enfermagem (COFEN) tem atuado na fiscalização das condições de trabalho, em especial acerca da falta dos EPIs, ressaltando que o déficit de profissionais decorrente dos afastamentos, poderá gerar um colapso no Sistema Único de Saúde. Em relação aos impactos na saúde mental, em conjunto com a Comissão Nacional de enfermagem em Saúde Mental, tem promovido oferta de atendimentos virtuais, que são direcionados aos profissionais de enfermagem que atuam na linha de frente de combate ao COVID-19 e ocorrem por meio de uma plataforma disponível de forma intermitente. ${ }^{17}$

Entretanto, tal situação não é restrita aos profissionais de enfermagem. Em pesquisa realizada com médicos, observou-se que o apoio logístico foi um fator associado ao bem-estar mental de profissionais que atuam na linha de frente do combate a pandemia. Para os autores, a sobrecarga de trabalho associada precariedade do ambiente de trabalho, no que diz respeito à insegurança e à escassez de EPIs, pode aumentar a percepção de risco. E em decorrência disso, ao aumento do medo de contagio e de exposição de familiares, refletindo na presença de sentimentos negativos como desespero e culpa e falta de motivação para o trabalho. ${ }^{20}$

Segundo o Ministério da Saúde, até o dia 08 de julho de 2020 foram notificados 786.417 casos suspeitos e 173.400 casos confirmados em profissionais de saúde no Brasil, sendo os mais acometidos os técnicos e auxiliares de enfermagem $(34,4 \%)$ e os enfermeiros (14.8\%). Os técnicos e auxiliares de enfermagem também registraram maior percentual de casos graves que necessitaram de internação (35,6\%), e maior percentual de óbitos (42,0\%) por SARS-CoV-2. ${ }^{21}$ A percepção de risco impacta negativamente os profissionais de saúde, em especial os de enfermagem, visto a alta disseminação e mortalidade do COVID-19 entre os mesmos.

Com relação aos fatores hereditários e biológicos, profissionais do sexo feminino tiveram resultados mais expressivos quantos aos sintomas de depressão, apresentando uma prevalência $62 \%$ maior que a observada no sexo masculino. Tal achado foi observado também no estudo de Lai et al. ${ }^{22}$, onde as enfermeiras relataram sintomas mais graves de ansiedade, depressão e angustia. Entretanto, a influência do sexo não foi observada quanto à prevalência de sintomas de ansiedade no presente estudo.

Em uma recente revisão sistemática e meta-análise, que buscou examinar as evidências dos efeitos da epidemia de COVID-19 na saúde mental dos trabalhadores da área da saúde, foi observado que as prevalências de depressão e ansiedade foram significativamente maiores entre os profissionais do sexo feminino. Segundo os autores, tal achado parece estar relacionado à diferença de sexo, já estabelecida na literatura, na prevalência sintomas destes transtornos mentais. Identificouse também, que os profissionais da equipe de enfermagem apresentaram as maiores taxas de depressão e de ansiedade dentre os trabalhadores de saúde. ${ }^{23}$

Sobre o efeito da raça/etnia, os profissionais de pele parda apresentaram uma prevalência $23 \%$ maior de sintomas que os brancos. Não foram observadas associações entre a pele amarela e preta, entretanto ressalta-se o número reduzido de profissionais com estas características no estudo. A ausência de indivíduos de pele preta pode estar associada ao acesso deficiente a educação, sabe-se que esta população tem maior probabilidade de sofrer os impactos da pandemia em virtude das negligências históricas, resultando maior vulnerabilidade social e econômica e menor acesso aos serviços, seja na condição de profissional de saúde ou usuário. ${ }^{24}$

Com relação aos fatores familiares, observou-se que morar com os pais ou irmãos é um fator associado a sintomas de depressão. Uma hipótese para tal achado, é que a convivência com idosos em seu círculo familiar aumenta a preocupação com a possível transmissão da doença em decorrência de sua 
exposição no trabalho. $\mathrm{O}$ fato de a idade ser um forte fator de risco para o óbito por COVID-19, pode ter gerado maior preocupação quanto à proteção e incentivo às medidas de distanciamento social nos profissionais que possuem idosos em sua convivência domiciliar. ${ }^{25,26}$

Liu et al. identificaram que os profissionais de saúde que não moram com familiares apresentaram maior risco para depressão, o que diverge dos achados na presente pesquisa. Apesar de os achados serem divergentes, ambos apresentam importantes implicações. Morar com familiares pode representar risco para a presença de sintomas, como o achado neste estudo, uma vez que o risco de contaminação e transmissão para seus entes queridos, associados aos efeitos que a doença provocou na vida destes profissionais, somados ao esgotamento físico e mental contribuem para o surgimento do sofrimento mental. ${ }^{27}$

Por outro lado, de acordo com Liu et al. ${ }^{6}$ a prevalência de sintomas de depressão entre os profissionais que não moram com os familiares pode ser justificada pelo apoio familiar como importante ponto de suporte emocional aos profissionais que atuam na linha de frente de combate à doença. $O$ presente estudo corrobora esses achados, visto que o hábito de conversar com familiares e amigos foi fator de proteção para sintomas graves de ansiedade e depressão.

O convívio familiar é um fator protetor, mas as intercorrências nesta relação são fatores contribuintes para os sintomas depressivos, aspectos próprios da profissão impactam no contato familiar, seja pelo cansaço e excesso de trabalho que prejudicam o convívio e o diálogo, pelas perdas familiares, ausência de suporte familiar e mesmo a carência deste contato, estes fatores favorecem o surgimento da depressão e do risco de suicídio. ${ }^{16}$ Portanto, a convivência familiar precisa ser analisada quanto a se constituir como fator de risco ou de proteção para o desenvolvimento de sintomas de depressão entre profissionais que atuam no atual cenário.

Além disso, a prática de atividades mente-corpo reduziu em $54 \%$ a prevalência de sintomas graves de ansiedade e a prática de atividades físicas reduziu em $36 \%$ a prevalência de sintomas graves de depressão entre profissionais de enfermagem. Assim, recomenda-se a adoção de hábitos saudáveis, com enfoque em atividades que visem o bem estar do corpo e da mente na prevenção e como adjuvante, considerando as evidências científicas sobre os benefícios e a inexistência de efeitos negativos para tais intervenções. ${ }^{28}$ Portanto, as atividades físicas, configuram-se como fatores de proteção para outras doenças crônicas não-transmissíveis.

\section{CONCLUSÕES E IMPLICAÇÕES PARA A PRÁTICA}

Observou-se uma alta prevalência de sintomas graves de ansiedade e depressão entre os profissionais de enfermagem que atuam nos serviços de média e alta complexidade durante a pandemia de COVID-19. Os fatores associados a uma prevalência mais acentuada de ambos os desfechos foram ter vínculo empregatício no setor privado, ter sintomas da Síndrome de Burnout e atuar em serviços sem estrutura para pandemia. Como fator de proteção para ambos os desfechos, ter o hábito de conversar com familiares e amigos. Tais resultados colaboram com os achados da literatura que demonstram o aumento do sofrimento mental entre os profissionais de enfermagem durante a pandemia.

Os resultados indicam que ações que visem à melhoria das condições de trabalho e que estimulem a prática de atividades físicas podem ser benéficas para o a manutenção e fortalecimento das condições de saúde mental dessa população. Vale ressaltar a importância de ações como as que o órgão representativo da profissão COFEN tem promovido - atendimentos virtuais; visto sua colaboração, seja na forma direta para estes profissionais, como indireta, para a população que é assistida através do SUS, evitando o risco de colapso no sistema por falta de profissionais, em virtude de sofrimentos psíquicos.

E compreendendo a importância destes profissionais nos serviços de saúde e considerando que muitos dos fatores contribuintes para os sofrimentos mentais estão relacionadas às condições de trabalho, sugere-se estratégias de promoção e valorização da profissão por meio de seus órgãos representativos e públicos.

Como limitações do estudo, destaca-se o delineamento metodológico desta pesquisa como web-surveys, que embora apresentem vantagens pela rapidez e possibilidade de coleta de dados em ambiente virtual, são passíveis de viés de seleção dos participantes que uma vez que sofre o efeito de "autosseleção", no qual o efeito do viés aumenta a prevalência do desfecho na população. ${ }^{29} \mathrm{As}$ medidas utilizadas para a redução do viés de seleção foram: seleção de profissionais-semente em todos os serviços de alta e média complexidade do estado para a divulgação do questionário eletrônico e o apoio do Conselho Regional de Enfermagem que divulgou o questionário para todos os profissionais inscritos.

\section{FINANCIAMENTO}

Coordenação de Aperfeiçoamento de Pessoal de Nível Superior (CAPES), código de financiamento 001; concessão de bolsa de pós-doutorado a Arthur de Almeida Medeiros [processo 88887.372306/2019-00], bolsa de mestrado a Katarina Márcia Rodrigues dos Santos, bolsa de doutorado a Maria Helena Rodrigues Galvão e Sávio Marcelino Gomes.

\section{CONTRIBUIÇÕES DOS AUTORES}

Desenho do estudo.. Maria Helena Rodrigues Galvão. Talita A raujo de Souza. Katarina Márcia Rodrigues dos Santos. Arthur de Almeida Medeiros. Isabelle Ribeiro Barbosa. Sávio Marcelino Gomes.

Coleta ou produção dos dados. Maria Helena Rodrigues Galvão. Sávio Marcelino Gomes. Talita Araujo de Souza. Katarina Márcia Rodrigues dos Santos. Arthur de Almeida Medeiros. Isabelle Ribeiro Barbosa

Análise de dados. Maria Helena Rodrigues Galvão. Sávio Marcelino Gomes. Talita Araujo de Souza. Katarina Márcia Rodrigues dos Santos. Arthur de Almeida Medeiros. Isabelle Ribeiro Barbosa 
Interpretação dos resultados. Maria Helena Rodrigues Galvão. Sávio Marcelino Gomes. Talita Araujo de Souza. Katarina Márcia Rodrigues dos Santos. Arthur de Almeida Medeiros. Isabelle Ribeiro Barbosa

Redação e revisão crítica do manuscrito. Maria Helena Rodrigues Galvão. Sávio Marcelino Gomes. Talita Araujo de Souza. Katarina Márcia Rodrigues dos Santos. Arthur de Almeida Medeiros. Isabelle Ribeiro Barbosa

Aprovação da versão final do artigo. Maria Helena Rodrigues Galvão. Sávio Marcelino Gomes. Talita Araujo de Souza. Katarina Márcia Rodrigues dos Santos. Arthur de Almeida Medeiros. Isabelle Ribeiro Barbosa

Responsabilidade por todos os aspectos do conteúdo e a integridade do artigo publicado. Maria Helena Rodrigues Galvão. Sávio Marcelino Gomes. Talita Araujo de Souza. Katarina Márcia Rodrigues dos Santos. Arthur de Almeida Medeiros. Isabelle Ribeiro Barbosa.

\section{EDITOR ASSOCIADO}

\section{Antonio Jose Almeida Filho}

\section{REFERÊNCIAS}

1. Huang Y, Zhao N. Generalized anxiety disorder, depressive symptoms and sleep quality during COVID-19 outbreak in China: a web-based cross-sectional survey. Psychiatry Res. 2020;288:112954. http://dx.doi. org/10.1016/j.psychres.2020.112954. PMid:32325383.

2. Wang D, Hu B, Hu C, Zhu F, Liu X, Zhang J et al. Clinical characteristics of 138 Hospitalized Patients with 2019 Novel Coronavirus-Infected Pneumonia in Wuhan, China. JAMA. 2020;323(11):1061-9. http://dx.doi. org/10.1001/jama.2020.1585. PMid:32031570.

3. World Health Organization. Coronavirus disease (COVID-19) [Internet] Geneva:WHO; 2020 [citado 2020 ago 8]. Disponível em: https://www. who.int/emergencies/diseases/novel-coronavirus-2019

4. Ministério da Saúde (BR). Coronavírus Brasil [Internet]. Geneva:WHO 2020 [citado 2020 ago 8]. Disponível em: https://covid.saude.gov.br/

5. Bhagavathula AS, Aldhaleei WA, Rahmani J, Mahabadi MA, Bandari DK. Knowledge and Perceptions of COVID-19 Among Health Care Workers: Cross-Sectional Study. JMIR Public Health Surveill. 2020;6(2):e19160. http://dx.doi.org/10.2196/19160. PMid:32320381.

6. Liu X, Kakade M, Fuller CJ, Fan B, Fang Y, Kong J et al. Depression after exposure to stressful events: lessons learned from the severe acute respiratory syndrome epidemic. Compr Psychiatry. 2012;53(1):15-23. http://dx.doi.org/10.1016/j.comppsych.2011.02.003. PMid:21489421.

7. Lung FW, LuYC, Chang YY, Shu BC. Mental symptoms in different health professionals during the SARS Attack: a follow-up study. Psychiatr Q. 2009;80(2):107-16. http://dx.doi.org/10.1007/s11126-009-9095-5. PMid:19247834.

8. Wu P, Fang Y, Guan Z, Fan B, Kong J, Yao Z et al. The psychological impact of the SARS epidemic on hospital employees in China: exposure, risk perception, and altruistic acceptance of risk. Can J Psychiatry. 2009;54(5):302-11. http://dx.doi.org/10.1177/070674370905400504. PMid:19497162.

9. Huang J, Liu F, Teng Z, Chen J, Zhao J, Wang X et al. Care for the psychological status of frontline medical staff fighting against Coronavirus Disease 2019 (COVID-19). Clin Infect Dis. 2020;ciaa385. https://doi. org/10.1093/cid/ciaa385

10. Clarke AL, Stephens AF, Liao S, Byrne TJ, Gregory SD. Coping with COVID-19: ventilator splitting with differential driving pressures using standard hospital equipment. Anaesthesia. 2020 jul;75(7):872-80. http:// dx.doi.org/10.1111/anae.15078. PMid:32271942.

11. Rio Grande do Norte. Governo do Estado. Secretaria de Estado da Saúde Pública. Informações importantes para a população em geral e profissionais de saúde sobre o novo coronavírus [Internet]. Natal:
Secretaria de Estado da Saúde Pública; 2020 [citado 2020 ago 8] Disponível em: https://portalcovid19.saude.rn.gov.br

12. Sant'Ana SMS. Ansiedade, depressão e qualidade de vida no trabalho em enfermeiros de hospitais públicos de médio e grande porte no município de Aracaju [tese]. Lagarto: Universidade Federal de Sergipe 2016 [citado 2020 ago 8]. Disponível em: https://ri.ufs.br/bitstream/ riufs/8009/2/SILVIA_MARIA_DA_SILVA_SANT\%e2\%80\%99ANA.pdf

13. Santos IS, Tavares BF, Munhoz TN, Almeida LSP, Silva NTB Tams BD et al. Sensibilidade e especificidade do Patient Health Questionnaire-9 (PHQ-9) entre adultos da população geral. Cad Saude Publica. 2013;29(8):1533-43. http://dx.doi.org/10.1590/S0102311X2013001200006. PMid:24005919.

14. Moreno AL, DeSousa DA, Souza AMFLP, Manfro GG, Salum GA Koller $\mathrm{SH}$ et al. Factor structure, reliability, and item parameters of the Brazilian-Portuguese Version of the GAD-7 Questionnaire. Temas Psicol 2016;24(1):367-76. http://dx.doi.org/10.9788/TP2016.1-25.

15. Que J, Shi L, Deng J, Liu J, Zhang L, Wu S et al. Psychological impact of the COVID-19 pandemic on healthcare workers: a cross-sectional study in China. General Psychiatry. 2020;33(3):e100259. http://dx.doi. org/10.1136/gpsych-2020-100259. PMid:32596640.

16. Silva DSD, Tavares NVS, Alexandre ARG, Freitas DA, Brêda MZ Albuquerque MCS et al. Depressão e risco de suicídio entre profissionais de Enfermagem: revisão integrativa. Rev Esc Enferm USP. 2015;49(6):102736. PMid:27419688.

17. Humerez DC, OhI RIB, Silva MCN. Saúde mental dos profissionais de enfermagem do Brasil no contexto da pandemia Covid-19: ação do Conselho Federal de Enfermagem. Cogitare enferm. 2020;25:e74115 http://dx.doi.org/10.5380/ce.v25i0.74115.

18. Ribeiro EG, Souza EL, Nogueira JO, Eler R. Saúde mental na perspectiva do enfrentamento à COVID-19: manejo das consequências relacionadas ao isolamento social. Rev Enfermagem e Saúde Coletiva. 2020;5(1):47-57.

19. Sousa OF, Cardoso N, Bezerra A, Pereira C, Nascimento G. Fatores relacionados ao adoecimento psicológico dos profissionais da equipe de enfermagem. Journal of Health Connections. 2020;9(2):24-44.

20. Faro A, Bahiano MA, Nakano TC, Reis C, Silva BFP, Vitti LS. COVID-19 e saúde mental: a emergência do cuidado. Estud. psicol. (Campinas). 37:e200074. https://doi.org/10.1590/1982-0275202037e200074.

21. Elbay RY, Kurtulmuş A, Arpacıoğlu S, Karadere E. Depression, anxiety, stress levels of physicians and associated factors in Covid-19 pandemics. Psychiatry Res. 2020;290:113130. http://dx.doi.org/10.1016/j. psychres.2020.113130. PMid:32497969.

22. Ministério da Saúde (BR). Boletim Epidemiológico Especial Doença pelo Coronavírus COVID-19 [Internet]. Brasília: Ministério da Saúde;2020 [citado 2020 ago 8]. Disponível em: http://saude.gov.br/images/pdf/2020/July/08/ Boletim-epidemiologico-COVID-21-corrigido-13h35.pdf

23. Lai J, Ma S, Wang $\mathrm{Y}$, Cai Z, Hu J, Wei $\mathrm{N}$ et al. Factors associated with mental health outcomes among health careworkers exposed to Coronavirus Disease 2019. JAMA Netw Open. 2020;3(3):e203976. http://dx.doi.org/10.1001/jamanetworkopen.2020.3976. PMid:32202646.

24. Pappa S, Ntella V, Giannakas T, Giannakoulis VG, Papoutsi E, Katsaounou P. Prevalence of depression, anxiety, and insomnia among healthcare workers during the COVID-19 pandemic: a systematic review and metaanalysis. Brain Behav Immun. 2020;88:901-7. http://dx.doi.org/10.1016/j. bbi.2020.05.026. PMid:32437915.

25. Goes EF, Ramos DO, Ferreira AJF. Desigualdades raciais em saúde e a pandemia da Covid-19. Trab Educ Saúde. 2020;18(3):e00278110. http://dx.doi.org/10.1590/1981-7746-sol00278.

26. Leão LRB, Ferreira VHS, Faustino AM. O idoso e a pandemia do Covid-19: uma análise de artigos publicados em jornais. Braz. J. of Develop. 2020;6(7):45123-42. http://dx.doi.org/10.34117/bjdv6n7-218.

27. Lima KC, Nunes VMA, Rocha NSPD, Rocha PM, Andrade I, Uchoa SAC, et al. A pessoa idosa domiciliada sob distanciamento social: possibilidades de enfrentamento à Covid-19. Rev. bras. geriatr. gerontol. 2020;23(2):e200092. https://doi.org/10.1590/1981 22562020023.200092

28. Prado AD, Peixoto $B C$, Silva AMB, Scalia LAM. A saúde mental dos profissionais de saúde frente à pandemia do COVID-19: uma revisão integrativa. Rev Eletrônica Acervo Saúde. 46:e4128. https://doi. org/10.25248/reas.e4128.2020.

29. Boni RB. Websurveys nos tempos de COVID-19. Cad Saude Publica 2020;36(7):e00155820. http://dx.doi.org/10.1590/0102-311x00155820. PMid:32638874. 